\title{
Vérité et dictature de l'étonnement, pour une éthique de la communication
}

Patrick Lamarque

\section{(2) OpenEdition}

1 Journals

Édition électronique

URL : http://journals.openedition.org/communicationorganisation/1595

DOI : 10.4000/communicationorganisation.1595

ISSN : $1775-3546$

Éditeur

Presses universitaires de Bordeaux

Édition imprimée

Date de publication : 1 novembre 1992

ISSN : 1168-5549

Référence électronique

Patrick Lamarque, "Vérité et dictature de l'étonnement, pour une éthique de la communication », Communication et organisation [En ligne], 2 | 1992, mis en ligne le 26 mars 2012, consulté le 01 mai 2019. URL : http://journals.openedition.org/communicationorganisation/1595 ; DOI : 10.4000/ communicationorganisation. 1595

Ce document a été généré automatiquement le 1 mai 2019.

(c) Presses universitaires de Bordeaux 


\title{
Vérité et dictature de l'étonnement, pour une éthique de la communication
}

\author{
Patrick Lamarque
}

1 L'un des paradoxes de l'époque réside dans le retour massif du symbolique, à travers l'organisation de médias de masses en système autonome, générant sa propre logique et comme fasciné par lui-même au point de transformer progressivement le jeu social en jeu de rôles. À lui seul, le mot grec qui signifie l'image dit clairement ce que nous adulons : eidôlon, l'idole.

2 Pour moi, l'un des enjeux majeurs de notre société, auquel volens nolens nous nous trouvons confrontés, à la fois comme professionnels de la communication et comme citoyens-cathodiques, c'est celui de la Vérité.

3 Non pas la vérité la plus simple, celle de Pinocchio qui fait s'allonger le nez du menteur. Celle-là est de toutes les époques et le menteur constitue un des types humains avec lesquels il faut apprendre à vivre. J'entends non seulement en société mais aussi en soimême, c'est-à-dire avec le menteur qui respire en nous.

4 Engager ainsi cette réflexion sur la Vérité par un éloge du mensonge - qui représente en même temps un aveu ! - mais chacun mesure combien mensonge et bonheur, mensonge et plaisir se trouvent liés par une profonde complicité. Imaginer, c'est mentir en inventant un réel virtuel ; vivre en société aussi, exige de mentir - songeons un instant au terrible dilemme du médecin face au malade incurable... Comme l'avoue clairement Dietrich Bonhoeffer ${ }^{1}$ "parler en vérité » impose de se faire très précautionneux, parfois même de trahir le réel évident au profit de la vérité subjective qui nous relie à notre interlocuteur.

D'où vient donc ce mystère qui fait qu'entre Réel et Vérité il n'y a pas totale homothétie et que le discours de vérité doit slalomer entre monde et mensonge ? Il vient de ce que le monde n'est pas réel, au sens où, inévitablement, dans l'expérience humaine il ne correspond pas à une « réalité objective » mais à une réalité perçue. Il vient également 
de ce que cette expérience du monde s'investit dans la relation à l'Autre. Voilà l'essentiel : l'Autre.

6 Il ne peut y avoir d'éthique, c'est-à-dire de pratique correcte ou de "mœurs bonnes ", pour parler comme Aristote ${ }^{2}$ sans qu'entre l'Autre, le monde et moi, la Vérité viennent établir la crédibilité de notre relation dialogique. Elle n'y est pas un promontoire isolé au centre de l'espace comme le nez au milieu du visage, mais une condition absolue de possibilité, à la fois pour notre existence au monde et pour notre communication avec l'Autre, notre « intersubjectivité » comme dirait Jùrgen Habermas ${ }^{3}$. À ce titre, l'exigence de Vérité constitue un des éléments indispensables à ce que John RAWLS appelle la « coopération sociale $»^{4}$. Faute de poursuivre la Vérité comme visée axiologique, il n'est pas de société vivable car ne s'y rencontre pas ce minimum de confiance sans lequel aucun lien n'est possible.

7 Pensons un instant à la difficulté qu'il y a à vivre - non pas agir, mais bien vivre - sans adhérer un minimum à cette idée simple que le discours rend compte d'une réalité du monde ou d'une vérité de l'être? Comment deux ennemis peuvent-ils entamer des négociations si n'existe pas ce lien de base ? Comment un individu peut-il éviter de glisser dans la folie s'il est radicalement objecteur à ses propres mots, donc à sa propre conscience? «Le silence intérieur est bruissant de paroles », écrivait Bachelard mais si mes propres paroles ne sont pour moi qu'un bruit confus, il n'est plus aucune conscience possible.

cette vérité du mot (et de l'image aussi) comme véhicule du Sens, notre société est en train de la mettre à mal à travers un double mouvement de dé-réalisation du Réel d'une part, et de dé-crédibilisation du Vrai d'autre part.

9 Il faut bien mesurer ce mouvement historique qui nous entrâne d'une dé-matérialisation à une dé-réalisation progressive du monde.

10 Ainsi de la monnaie. Lorsqu'elle fut inventée, elle n'avait de sens qu'à proportion du poids de métal précieux qu'elle contenait. Elle formait une réalité en soi. Puis, à partir de François Ier, pour financer les guerres, les gouvernements ont appris à «rogner les écus ", donc à introduire un écart entre valeur réelle et valeur faciale. Lorsque les assignats sont apparus, c'est-à-dire une figuration de la valeur sur du papier à cours forcé, l'argent s'est soudain transformé en un bien purement fiduciaire - fondé sur la confiance -. Depuis, ce sont les banques qui créent de la monnaie : plus elles prêtent, plus il y a de l'argent en circulation. Or ces prêts ne sont pas conditionnés par l'existence préalable de stocks monétaires en caisse, mais par un simple jeu d'écritures, uniquement fondé sur la décision de prêter. Ainsi l'argent s'est, peu à peu, dé-matérialisé.

11 Il en va de même pour l'ensemble de l'économie, dont les facteurs de croissance les plus déterminants ne sont plus désormais les facteurs le plus matériels (capital et travail) mais le niveau technologique et celui de l'éducation. En quittant l'économie-hard pour accéder à l'économie-soft, inévitablement c'est le monde qui se dé-matérialisé.

Quant à notre relation perceptive au Réel, il faut bien convenir que nous sommes entrés dans « la société du spectacle ", comme la nommait de façon prémonitoire Guy Debord, dès $1967^{5}$. Le Vrai fait place au vraisemblable et la Vérité à la véracité, c'est-à-dire à ce qui a l'air vrai. Rappelons nous la fin de l'année 1989 et Timisoara. Le monde entier tétanisé par les images du charnier roumain.

13 Un journaliste de feu la Cinq - le seul regard professionnel occidental présent sur place téléphone à sa rédaction : pour lui il n'y a pas eu une répression aussi massive qu'on le dit 
des manifestations des semaines précédentes. Il entend alors son rédacteur en chef lui rétorquer qu'il n'a rien compris, qu'il ne peut pas lui, quoique sur place, avoir raison à la fois contre les images et contre CNN, CBS, ABC, TP1, la RAI, A2, BBC, NHK..., bref contre ce qu'il faut aujourd'hui appeler «le monde entier».

Car le mythe platonicien de la caverne n'a jamais eu plus de sens qu'aujourd'hui. Les petites lucarnes à travers lesquelles nous nous faisons une certaine idée du réel génèrent un curieux phénomène optique : elles nous affublent d'une très longue focale qui nous fait voir le lointain aussi proche que le voisinage mais, tout en même temps, elles rétrécissent le champ de notre vision.

À cet effet de la technologie s'ajoute un phénomène d'une autre nature que j'appelle le syndrome de l'effet, ou la dictature de l'étonnement. Les médias, tous ensemble, sont entrés dans une concurrence d'autant plus sauvage que leur survie dépend pour une part déterminante de leurs rentrées publicitaires. De surcroît, cette compétition est planétaire, du fait de la généralisation des satellites (CNN nous investit). Pour conserver leur audience, ils se trouvent irrémédiablement contraints à nous accrocher, à nous séduire à longueur de programmes. Ils entrent alors dans une double course: à l'instantanéité d'abord - il faut aller toujours plus vite que les concurrents, au risque de moins vérifier les informations, d'être moins rigoureux - ; au spectacle ensuite - «fais fort, coco ", voilà la consigne de base de tous les rédacteurs en chef.

16 L'évolution fut brutale: dans les années cinquante et soixante, la consigne bien connue d'Hubert Beuvemery, le rédacteur en chef du Monde était : « Messieurs, faites chiant ». Il fallait avoir l'air austère pour signifier le sérieux. Aujourd'hui, il n'est pas un patron de presse économique qui ne répète à ses journalistes: «Messieurs, l'économie ça se raconte ». Avec cette invasion du narratif, l'information se trouve progressivement désinvestie par le Sens pour livrer la place au bruit. Et le bruit tue le Sens. Dans la conversation comme dans le monde.

17 Cette logique d'étonnement qui substitue le bruit à l'argument se répand dans la société tout entière, du fait du rôle polarisant du système médiatique dans l'espace public. Désormais, les règles en vigueur dans l'expression publicitaire s'étendent aux médias dans leur ensemble, pour gagner également la politique et le management. Les chefs d'entreprise, après les élus, deviennent des stars qui consacrent une part de plus en plus importante de leur temps à exister dans les médias.

Le constat est terrible : qui n'est pas régulièrement présent dans les médias n'existe pas. Il n'a pas de réalité !

19 Évidemment, nous, directeurs de la communication, nous nous trouvons au cœur du complot dont, ès fonctions, nous sommes des chevilles ouvrières. À l'interface de l'entreprise ou de l'organisation et du monde médiatique, nous le nourrissons d'images et de bruits. À l'intérieur de nos organisations, à travers la communication interne qui, va se professionnalisant, nous recourons de plus en plus aux règles de l'univers médiatique. Nous sommes donc parmi les conjurés le plus actifs du putsch de l'étonnement.

20 En même temps acteurs et spectateurs, nous nous situons parmi les marionnettistes tout en nous trouvant assis aux bancs du public. Si un jour se produit un crime communicationnel, nous pouvons en être soit les exécuteurs des basses œuvres, soit les victimes. À l'origine de la faute, ou citoyens trompés parmi les autres.

21 Une responsabilité majeure pèse donc sur nous, femmes et hommes de communication, dans tous les mâillons de la chaîne d'information. À force d'abuser des moyens du 
spectacle dans la transmission des faits, nous risquons de soumettre la perception du réel par le public aux mêmes règles que celles de la production d'imaginaire et, ce faisant, de contribuer à dé-crédibiliser les vecteurs de communication. Certes, il y a beau temps que le journal est affublé du sobriquet ironique, mais somme toute affectueux, de "petit menteur». Cependant, lorsque l'opinion aura bel et bien perdu confiance dans ses médias, elle ne sera pas loin d'avoir jeté tout lien social aux orties. Comment croira-t-elle en ceux qui la gouvernent si tous mentent? Comment s'engagera-t-elle réellement dans la vie professionnelle, comme on l'en supplie, si les dirigeants la manipulent? Comment le pacte social se main-tiendra-t-il si le discours public se trouve soudain dépourvu de toute crédibilité, quand il constitue la matière même de la relation intersubjective?

La relation entre le mot et le monde s'en trouvera minée de l'intérieur justifiant tous les scepticismes, tous les repliements. Les intérêts individuels seront l'unique mesure du bien et du mal, à l'exception de quelques solidarités limitées. Alors que nous espérons évoluer vers des sociétés élargies, nous serons entrés dans des micro-solidarités aux relents parfois tribaux ou claniques. Ce qui se passe en Yougoslavie est un exemple qui nous éclabousse d'évidence : à une logique ethnique s'agrège une pratique de bandes armées se taillant des territoires à la mesure de leurs forces et, dans les interstices, les "snipers ", c'est-à-dire les loups solitaires.

Si nous perdons le sens du Vrai, nous perdons aussi celui du Mot. Alors le pacte social civilisé, fondé sur la diffusion démocratique des informations et l'appropriation du réel par le plus grand nombre s'effondre, par disparition du lien de confiance. Et nous nous enfonçons dans l'obscurité d'un nouveau moyen-âge. Puisque le système communicationnel se trouve désormais au cœur du lien social, il lui échoit des responsabilités à la mesure de son rôle, et les communicateurs se doivent à une éthique exigeante qui dessine une vision claire de ce qui est essentiel.

Et, à mes yeux, l'essentiel c'est qu'il n'est pas de société viable sans une exigence forte de vérité.

BIBLIOGRAPHIE

ARISTOTE, Éthique de Nicomaque, Paris, Garnier-Flammarion nº 43,1965.

BONHOEFFER (Dietrich), Éthique, Genève, Labor et Fides, 1989.

DEBORD (Guy), La société du spectacle, Paris, Buchet-Chastel, 1967.

HABERMAS (Jürgen), Morale et communication, conscience morale et activité communicationnelle, Paris, Éd. du Cerf, 1986.

RAWLS (John), Théorie de la justice, Paris, Éd. du Seuil, 1987.

RICOEUR (Paul), Soi-même comme un autre, coll. l'ordre philosophique, Paris, Éd. du Seuil, 1990.

SFEZ (Lucien), Critique de la communication, Coll. la couleur des idées, Paris, Éd. du Seuil, nouvelle éd. 1990. 
WATZLAWICK (Paul), L'invention de la réalité, Paris, Éd. du Seuil, 1988.

\section{NOTES}

1. Éthique, Genève. Labor et Fides,1989

2. Éthique de Nicomaque. Paris, Garnier-Flammarion $\mathrm{N}^{\circ} 43,1965$

3. Moralee et communication, conscience morale et activité communicationnelle. Paris, Ed. du Cerf. 1986

4. Théorie de la justice, Paris. Éd. du Seuil, 1987

5. Paris, Buchet-Chastel

\section{RÉSUMÉS}

L'exigence de vérité représente la condition de possibilité d'une coopération sociale. Or elle s'inscrit dans un mouvement de déréalisation du réel, lié au triomphe de la véracité sur la vérité. L'urgence, la recherche de l'effet dans le traitement de l'information, la prépondérance du narratif sur le discursif Induisent un reflux $d u$ Sens au profit du bruit dans le système médiatique. Le risque est alors celui d'une perte de confiance radicale dans le principal lieu actuel du discours public.

\section{AUTEUR}

\section{PATRICK LAMARQUE}

Directeur de communication à la Délégation Générale pour l'Armement après avoir coordonné la communication gouvernementale au Service d'Information et de Diffusion du Premier ministre, Patrick Lamarque, ancien élève à l'ENA, consacre une part importante de son temps à l'enseignement et à la recherche. Méthodologue, il a développé des techniques qualitatives d'analyse de l'efficacité des campagnes institutionnelles télévisées. Il s'apprête à publier un essai consacré à l'éthique dans l'espace public. 